\title{
Kognitiv und/oder epistemisch? Auf dem Weg zur epistemologischen Semantik ${ }^{1}$

\author{
Cognitive and/or epistemic?
} Towards the Epistemological Semantics
}

\begin{abstract}
When embarking on a journey towards the constitution of epistemological semantics one needs to describe clearly the key term 'epistemic' and differentiate between this term and the term 'cognitive', which has been used so far, but has in time become ambiguous. The author of this article does not present the history of using these terms. Instead he undertakes to clarify the term 'epistemic' in order to enable himself to operate on problems linked with making epistemological semantics legitimate, including the problem of using specialized neologisms.
\end{abstract}

KEYWORDS: epistemic, cognitive, term, epistemological semantics.

\section{PHILOSOPHISCHE VS. LINGUISTISCHE FRAGESTELLUNG}

Da der vorliegende Aufsatz als ein linguistischer Beitrag zur Semantik zu verstehen ist, wird hier prinzipiell von der philosophisch geprägten Tradition zur Epistemologie, d. h. von ihren Deutungen bzw. Definitionen abgesehen, die nicht nur in der Vergangenheit, etwa in der sog. historischen Epistemologie (detailliert dazu s. Skowronek 2009: 129, 134), sondern auch gegenwärtig in der sog. naturalistischen Epistemologie diskutiert werden

${ }^{1}$ Dieser Beitrag stellt eine Einleitung zum Text Ein Plädoyer für die epistemologische Semantik dar, der im nächsten Heft der Glottodidactica erscheinen wird. 
(vgl. u. a. Quine, 1969, 1981, 1995; Fumerton 1994; Plümacher 2004; Bock von Wülfingen 2010; Gabriel 2008; Moser 2012; Künne 2007). Ich distanziere mich außerdem von der autoepistemischen Logik etwa dieser bei Robert C. Moore (1983) oder Yoav Shoham (1987) und insbesondere von der philosophisch ausgerichteten epistemischen Semantik, vertreten $u$. a. von Edouard Machery (u. a. 2012) und Michael Devitt (2012a/b). Die Gründe sind verschieden: genannt seien in erster Linie die methodologischen und terminologischen. Plausible Gründe, warum bisherige philosophische Fragestellungen den linguistischen Forschungsgegenstand und die linguistische Zielsetzung verfehlen, liefert Sambor Grucza (2012) im Kapitel Sprache und Erkenntnis - Bisherige Konzepte für die Erfassung der kognitiven Funktionen der Sprache.

Das erste Problem, auf das man bei der Lektüre von Arbeiten der Sprachphilosophien stößt, ist die Unklarheit, ob sie sich mit der Erkenntnis als Tätigkeit oder der Erkenntnis als Hervorbringung der Tätigkeit befassen (...). Das zweite Problem liegt darin, dass, obgleich die Vertreter der Sprachphilosophie behaupten, sie interessieren sich für Erkenntnisakte und sich doch nur mit den Regeln der Annäherung an das wahre Wissen, die Wahrheit, befassen. Ein weiteres Problem liegt darin, dass ebenfalls nicht ganz klar ist, was das wahre Wissen (Wahrheit) ist, und was es nicht ist. Es ist nämlich schwer zu bestimmen, in welcher Bedeutung sie das Wort „Wissen" verwenden. Im Endergebnis bedeutet dies, dass, obgleich die Disziplin als „Erkenntnistheorie“ bezeichnet wird, sie sich in Wahrheit gar nicht mit dem Wissen, sondern mit einer Art des Wissens, mit dem wahren Wissen, d. h. der Wahrheit, befasst. Es ist jedoch außerordentlich wichtig, was die Vertreter der Erkenntnistheorie unmissverständlich betonen, dass die Überlegungen zur Sprache unbedingt mit den Fragen zu verbinden sind, die sich auf das Wissen beziehen. (S. Grucza 2012: 87-88)

Die konzeptuelle Inkongruenz der philosophischen Erkenntnistheorie und der Linguistik hinsichtlich der Erfassung des Forschungsgegenstandes, der Aufgaben und der Erkenntnisziele liegt auf der Hand und ist einfach nicht wegzudenken. Diese Inkongruenz inspiriert. Sie ,hilft' nämlich eine Frage formulieren, die in der Semantikforschung bisher selten gestellt wurde: die Frage nach der epistemischen Arbeit, und genauer, nach den Erkenntnisakten eines Menschen, der mit Blick auf die intendierenden Kommunikationsakte genötigt ist, einen neuen (Fach-)Ausdruck bilden und einführen zu müssen. Es handelt sich hier, kurz gesagt, um epistemische Faktoren, die die idiolektale Ausdrucks- und Bedeutungskonstitution motivieren und aktivieren. Diese Fragestellung ist für die epistemologisch ausgerichtete Semantik schlüssig und bedarf selbstverständlich empirischer Belege (vgl. hierzu Pawłowski 2015a, b, c). 


\section{UNKLARE GRENZE ZWISCHEN ,KOGNITIV' UND ,EPISTEMISCH'}

Nicht weniger wichtig scheint die Frage zu sein, wie der Ausdruck ,epistemisch' bisher gedeutet wurde. In der Diskussion darüber wird hier von den linguistischen Konzeptionen ausgegangen, in denen vor allem das (menschliche) Subjekt in den Vordergrund der Forschung gerückt wird. Ich greife dabei zuerst auf die 2008 und 2012 formulierten Thesen zur linguistischen Epistemologie von Dietrich Busse und den Anfang 2012 von Franciszek Grucza verfassten Beitrag Zum Gegenstand und zu den Aufgaben der Anthropozentrischen Linguistik und Kulturologie zurück.

Dietrich Busse zieht 2008 keine klare Grenze zwischen ,kognitiv` und ,epistemisch". "Kognitive“ und „epistemische Leistungen“ werden weder explizite gedeutet noch voneinander differenziert (2008c: 11). Unklar ist außerdem, genau auf welche Designate "kognitive/epistemische Prozesse“ und "sprachliche und epistemische Regeln“ genau referieren.

Der kognitiven Semantik geht es dagegen mehr um die Strukturen, die das verstehensermöglichende Wissen annimmt, und um die kognitiv-epistemischen Prozesse, die für die jeweils neu zu vollziehende Konstitution bzw. Realisierung von sprachzeichengestütztem Sinn eine notwendige Voraussetzung sind. (Busse 2008c: 16)

\section{Und weiterhin}

Jedes Feststellen der ,Bedeutung' eines Wortes, Satzes, Textausschnitts ist daher in einem gewissen Sinne implizit ,historisch', sofern es auf Bedeutungskonventionen (und andere sprachliche oder epistemische Regeln) zurückgreift. (Busse 2008c: 12)

Busse erläutert nicht, wie „kognitiv-epistemische Prozesse“ konzeptuell und terminologisch zu begreifen sind. ${ }^{2}$ Es ist schwer nachzuvollziehen, im welchem Verhältnis die Designate der Ausdrücke ,epistemisch` und ,kogni-

\footnotetext{
2 Das Problem der epistemischen Leistung beschäftigt Dietrich Busse bereits in seinem Aufsatz mit dem Titel Architekturen des Wissens - Zum Verhältnis von Semantik und Epistemologie. Hier verspürt man deutlich den Gedanken, demzufolge Wissensaktivierung, d. h. die Aktivierung der kognitiven Prozesse mit - wie zu erhoffen ist - neuen Erkenntnisakten in Bezug gebracht wird: „Im epistemischen Akt, im Moment der Wissensaktivierung lassen sich deren Bedingungen und Voraussetzungen nicht zugleich bewußt machen und würden sich in Konsequenz auch jeder explizierenden wissenschaftlichen Analyse entziehen." (ders. 2005: 49). Dies wird allem Anschein nach in seinem späteren Aufsatz bekräftigt: „Der kognitiven Semantik geht es dagegen mehr um die Strukturen, die das verstehensermöglichende Wissen annimmt, und um die kognitiv-epistemischen Prozesse, die für die jeweils neu zu vollziehende Konstitution bzw. Realisierung von sprachzeichengestütztem Sinn eine notwendige Voraussetzung sind." (ders. 2008c: 16).
} 
tiv ${ }^{\prime}$ zueinander stehen. Diese Insuffizienz kann man außerdem an anderen Stellen beobachten: "kognitive (epistemische) Leistungen“, "auf denselben kognitiven (epistemologischen) Prinzipien beruhen" oder "Gehalt menschlicher Kognitionsprozesse und Episteme" (vgl. ders. 2008c: 11, 16, 18, 26). Es ist hier vor allem unklar, wie der Ausdruck ,epistemisch' gedeutet wird. Die früheren Aufsätze von Busse lassen sogar suggerieren, dass „epistemische Prozesse“, "Denkbewegungen“ also, wie zu deuten ist, ganz konkrete mentale (ggf. unbewusste) Erkenntnisakte, aus den kognitiven Akten im engeren Sinne, ausgeschlossen werden. In der 2012 erschienen Monografie mit dem Titel Frame-Semantik äußert sich Busse zu diesem Problem in einer expliziten Stellungnahme. Aus dieser geht hervor, dass der "epistemische Wissensrahmen " 3 als eine kognitive Struktur im Wissen aufzufassen ist.

Als Modell für die Beschreibung der Strukturen, Ebenen und Elemente des verstehensrelevanten (semantisch relevanten) Wissens ist das Frame-Modell angeboten worden. Im Sinne der Entwicklung eines Modells der linguistischen Semantik mit dem beschriebenen Zuschnitt kommt dafür nur ein Verständnis der Frames als Strukturen im Wissen, als ,epistemische Frames' in Frage. Üblicherweise werden solche Aspekte nicht mir dem Ausdruck ,epistemisch', sondern dem Ausdruck ,kognitiv ${ }^{\prime}$ belegt. In diesem Sinne liegt einer epistemologisch reflektierten Semantik notwendigerweise ein Modell ,kognitiver Frames' zugrunde. Da die übliche Verwendung des Ausdrucks ,kognitiv' aber Aspekte einschließt, auf die es hier (im Modell der linguistischen Semantik) zunächst weniger ankommt (...), und die semantische Analyse stärker auf die ,Inhalte' selbst (eben das verstehensrelevante Wissen) in seinen

3 "Ich habe (...) den Begriff ,Wissensrahmen' verwendet (in Anlehnung an die Begriffe ,scenes', ,skripts', ,schema', ,frames' etc. in der Textlinguistik); dieser Begriff muss zu der vorangegangenen Differenzierung der epistemischen Voraussetzungen des Textverstehens in Beziehung gesetzt werden. Ich verstehe unter den verstehensrelevanten Wissensrahmen konkrete (d. h. themenspezifische) Agglomerationen von Wissenselementen, welche die verschiedenen Aspekte des formalen Modells der Wissensdifferenzierung in unterschiedlicher Weise verwirklichen. Die vorgenommene Differenzierung von Wissensebenen, Wissenstypen und Wissensmodi sollte als formale Klassifizierung aufgefasst werden. Dagegen können Wissensrahmen konkret-inhaltlich in ähnlicher Weise geschrieben werden, wie etwa Textbedeutungen. Da wir das Textverstehen als die Fähigkeit des In-Beziehung-Setzens von Ausdruckselementen zu Wissenselementen bezeichnet haben, ist die Beschreibung der verstehensrelevanten, bzw. genauer: Verstehen bzw. Textbedeutung überhaupt ausmachenden, Wissenselemente, und damit die Beschreibung ihrer themenspezifischen Komplexierungen in Wissensrahmen, Teil der semantischen oder interpretativen Tätigkeit selbst. Wie weit ein Wissensrahmen in der konkreten Textanalyse oder -interpretation gezogen wird, ist dann eine Frage der Begrenzung oder Ausweitung des analytisch/interpretativen Zugriffs. Jede Aufzählung und Beschreibung verstehensrelevanten Wissens (also auch die Beschreibung und Begrenzung der verstehensrelevanten Wissensrahmen) ist das Herstellen eines analytischen bzw. interpretativen Konstrukts, und daher mit allen Unwägbarkeiten und Unsicherheiten behaftet, wie jede interpretative Tätigkeit.“ (Busse, Textinterpretation 1992: 162 f., nach Busse 2012: 524 f., zur Deutung von Wissensrahmen vgl. auch Busse 2008d) 
Strukturen, Ebenen und Elementen) zielt, fasse ich diese Frames als ,epistemische Frames' oder eben ,Wissensrahmen' auf. (Busse 2012: 535 f.)

Die epistemologisch orientierte Reflexion zur Semantik soll demnach notwendigerweise auch die kognitiven Relationen in Frames und die sich im (epistemischen) (Wissens-)Rahmen konstituierenden Strukturen des verstehensrelevanten Wissens miteinbeziehen. Mit Recht werden hier m. E. epistemische Frames als kognitive Wissensstrukturen aufgefasst. Diese sind ja schließlich das Ergebnis der epistemischen Leistung eines Individuums oder ggf. das Ergebnis seiner bewusst ausgeführten Erkenntnisakte. Dadurch wird jedoch die konzeptuelle Vagheit, die darin besteht, dass kognitive Frames mit den epistemischen Frames quasi gleichgesetzt werden, noch lange nicht behoben. Die explizite Bezugnahme auf ,epistemisch" - „das auf vorgängige Erkenntnisakte zurückgehende Wissen“ - und auf den Ausdruck ,kognitiv', dem Erkenntnisakte schlechthin beigemessen werden, lässt das Problem auch nicht lösen.

Ich verwende (...) häufig die Doppelform ,kognitiv/epistemisch' (oder umgekehrt), da beide Begriffe zwar etwas bezeichnen, das eng zusammenhängt, sie aber keineswegs synonym sind. Während in "kognitiv' eher der Erkenntnisakt fokussiert wird, betont ,epistemisch' eher das auf vorgängige Erkenntnisakte zurückgehende Wissen als solches. Beide Begriffe repräsentieren also das, was Grice einmal als , act-objectambiguity' charakterisiert hat: einen (hier: geistigen) Akt und sein Ergebnis. (Busse 2012: 23, Anm. 1)

\section{EPISTEMISCHE VERANLAGUNG DES MENSCHEN}

Franciszek Grucza erläutert das Problem, epistemisch und/oder kognitiv` nicht. Er leistet dafür einen wesentlichen Beitrag zur besseren Erfassung der epistemischen Arbeit, unter anderem, zur Erkenntnishandlung und zu den Erkenntnisakten, denen eindeutig kognitive Attribute zugeschrieben werden.

Ich verwende nicht nur die Ausdrücke „Erkenntnishandlung(en)“ und „kognitive Handlungen“, sondern auch die Ausdrücke „kognitive Handlungsprozesse“ und „kognitive Erkenntnisprozesse“ im Sinne bestimmter kollektiver Bezeichnung. Die ersteren, indem ich mit ihrer Hilfe sowohl Handlungsakte als auch Handlungsprozesse hervorhebe, und die letzteren, weil ich mit ihrer Hilfe sowohl entsprechende Ketten von Erkenntnisakten hervorhebe, die binnen eines Zeitverlaufes durch ein und dasselbe Subjekt als auch solche, die durch verschiedene Subjekte zum Zweck der Bereicherung seines/ihres Wissens über denselben Gegenstand realisiert werden. 
Beruflich ausgeführte kognitive Handlungen hebe ich mit Hilfe des Ausdruckes „Erkenntnisarbeit“" hervor. (F. Grucza 2012b: 299)

Menschliche Erkenntnisaktivitäten, seien es bewusste oder unbewusste, seien es gezielte oder ungezielte, sind nach F. Grucza prinzipiell auf die mentale Sphäre eines konkreten Individuums zurückzuführen: „(...) im Falle eines jeden Erkenntnisaktes handelt es sich immer um eine Erkenntnishandlung, die von einem bestimmten (konkreten) individuellen Subjekt ausgeführt wird." (F. Grucza 2012b: 312). Franciszek Grucza trennt zwischen den "sinnlichen und mentalen Erkenntnisaktivitäten“, die auf dem "sinnlichen und/oder mentalen Potenzial“ sowie zwischen den angeborenen und „von ihm [Lebewesen] generierten (erzeugten oder nacherzeugten) Fähigkeiten“ basieren.

Die Erkenntnisaktivitäten der Lebewesen gliedere ich primär aufgrund eines teleologischen Kriteriums und nicht aufgrund methodologischer Kriterien, und schon gar nicht aufgrund eines inhärenten Merkmals (seiner substantiellen Konstituenten) dieser Aktivitäten heraus. (...). Hier sei lediglich hinzugefügt, dass ich die logische Summe der Erkenntnisaktivitäten aller Lebewesen (darunter auch die logische Summe der Erkenntnisaktivitäten aller Menschen) in zwei Hauptkategorien - in die Kategorie sinnlicher und die Kategorie mentaler Erkenntnisaktivitäten - einteile. (F. Grucza 2012b: 295, vgl. auch 296)

Die letztere Unterscheidung zwischen den sinnlichen und den mentalen Erkenntnisaktivitäten will in dem hier besprochenen Aspekt besonders hervorgehoben werden, denn damit wird definitiv zum Ausdruck gebracht, dass sinnliche Aktivitäten, wie das Sehen, Hören, Tasten, Riechen usw., nicht automatisch und nicht notwendigerweise kognitive Erkenntnisakte sind und/oder letztere initiieren müssen. Dem Sehen, Hören, Tasten, Riechen liegen zwar kognitive Prozesse zugrunde, fundiert auf konkreten/einmaligen neurobiologisch organisierten Spikes, d. h. auf bestimmten Spitzenpotenzialen, Aktionspotenzialen oder auf chemischer Signalgebung im Gehirn - kognitive Prozesse dieser Art sind das Ergebnis bloßer RezeptorenAktivitäten -, so ist die Wissenskonstitution/Signalspeicherung bekanntlich noch lange nicht gesichert (!) Schließlich sehen, hören, tasten oder riechen wir nicht bloß mit unseren Augen, Ohren, Fingern und riechen auch nicht mit der Nase allein. Das Erkenntnis-Ergebnis wird erst infolge eines Erkenntnis-Aktes, dem die Wissenserlangung durch ein konkretes Subjekt folgt, realisiert.

Außerdem sei schon an dieser Stelle angedeutet, dass ich infolge der dargelegten Unterscheidungen die Bedeutungen der Ausdrücke „Ergebnis einer Erkenntnishand- 
lung“, „Ergebnis eines Erkenntnisaktes“ und „Ergebnis eines Erkenntnisprozesses“ keinesfalls als äquivalente Bedeutungen behandle, jedoch mit ihrer Hilfe jeweils auf ein bestimmtes Wissensquantum verweise, das das (individuelle bzw. kollektive) Subjekt infolge der Ausführung der Handlung (des Aktes oder Prozesses) dazugewonnen hat. Mit anderen Worten: Unter „Erkenntnisergebnis“ verstehe ich in jedem Fall ein infolge der Realisierung des Erkenntnisaktes erlangtes Wissen seines Subjektes. (F. Grucza 2012b: 312)

Daraus ist $\mathrm{zu}$ folgten, dass Wissenselemente/Engramme - wie ich sie seit einigen Jahren gemäß der Gedächtnisforschung zu bezeichnen pflege ,wortwörtlich' das Ergebnis - oder wie Franciszek Grucza wohl sagen würde, das wirkliche Ergebnis der epistemischen Leistung eines konkreten Individuums sind. Das Erkenntnispotenzial, die Erkenntnisfähigkeit und die Fähigkeit zu Entwicklung dieses Erkenntnispotenzials sind seine (konstitutiven) Eigenschaften und werden von Franciszek Grucza mit dem Ausdruck "epistemische Merkmale/Faktoren“ bezeichnet (vgl. F. Grucza 2012b: 297).

\section{ETWAS NEUES IN DER KOGNITION}

Es bestehen keine Zweifel, dass die menschliche Erkenntnisfähigkeit genetisch veranlagt ist. In dieser Hinsicht sollen die Designate von ,epistemisch' wohl unmissverständlich als bestimmte Eigenschaften der menschlichen Konstitution, sozusagen seiner anthropologischen Ausstattung, aufgefasst werden. Unumstritten bleibt wohl auch die Frage, warum diese Eigenschaften vor allem auf die kognitive Sphäre zurückzuführen sind und warum sie individuell und jeweils vom Neuen geformt werden. Dagegen nicht evident ist die Tatsache, wie dieses Neue auf der Ebene der menschlichen Kognition konzeptuell und terminologisch erfasst werden kann und soll, wie/wodurch dieses (kognitive) Neue geformt wird und wie/aufgrund welcher Mechanismen dieses (kognitive) Neue zum Ausdruck gebracht werden kann. Nicht evident bleibt außerdem,

- dass mit diesem Neuen das neue Wissen identifiziert wird,

- dass das Wissen erkenntnisfördernd sein kann,

- dass das neue Wissen die (reine) Erkenntnis ist und

- dass die neue Erkenntnis nicht selten Termini oder (Fach-)Neologismen erfordert, die interne Eigenkommunikation und erst nach Bedarf externe allgemeine- bzw. fachorientierte Kommunikation konstituieren, viel mehr, diese sogar voraussetzen.

Diese Annahmen gelten m. E. vor allem für die Konstitution von (Fach-)Neologismen. Damit mag zuerst nur eine grobe Ausgangsplattform 
geschafft sein, die hier prinzipiell dazu dient, um bei der epistemologisch ausgerichteten Reflexion zur idiolektalen Ausdrucks- und Bedeutungskonstitution grundlegende Konzepte und einige plausible Lösungen zur empirischen Analyse von Fachtexten festzuhalten.

\section{HERKUNFT DES AUSDRUCKS ,EPISTEMISCH`}

Um zu verstehen, wodurch sich der epistemologische vom kognitivistischen Ansatz zur Semantik abhebt, soll vor allem geklärt werden, wie der Terminus, epistemisch' zu verstehen ist und warum es eigentlich vom Belang ist, ihn mit ,kognitiv' zu kontrastieren. Bei der Deutung des Terminus ,epistemisch' gehe ich sozusagen pflichttreu zunächst einmal von der Herkunft des Ausdrucks, ,epistemisch' aus. ,Epistemisch' geht auf das griechi-

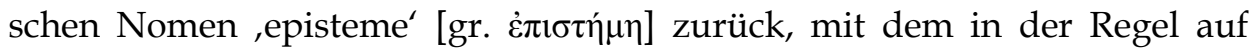
(wissenschaftliches) „Wissen“ und gewöhnlich auch auf „Erkenntnis“ verwiesen wird. Im griechisch-polnischen Sprachwörterbuch wird beim Lemma $\dot{\varepsilon} \pi ા \tau \eta \dot{\mu \eta}$ jedoch zuallererst „umiejętnośćc [dt. Fertigkeit], „znajomość" [dt. Kenntnis/Können], „wprawa“ [dt. Geschicklichkeit], „biegłość“ [dt. Gewandtheit], „zręczność“ [dt. Handfertigkeit] und erst dann „wiedza“ [dt.

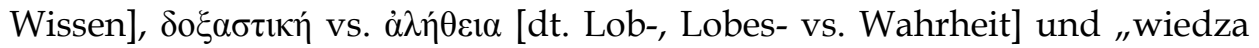
naukowa" $\tau \dot{\chi} \chi v \eta$ [dt. wissenschaftliches Wissen] angegeben (vgl. SłGP 1960). ${ }^{4}$ Geschichtlich gesehen ist $\dot{\varepsilon} \pi \_\tau \dot{\mu} \mu \eta$ eine Ableitung vom verbum deponens

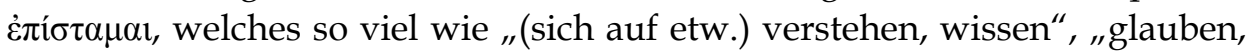

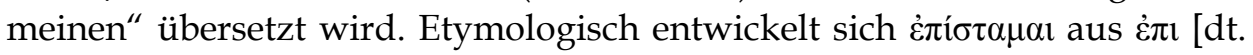
vor (etwas)] und altgr. ǐ $\sigma \tau \mu \iota$ bzw. dor. í $\sigma \alpha \mu \mu \iota$ [dt. (sich) stellen, stehen, an-

${ }^{4}$ Wissen und Erkenntnis wurde vor Sokrates über Plato und Aristoteles der bloßen Sinneswahrnehmung eindeutig entgegengesetzt. Episteme „(...) d. h. das spekulative Denken des einzelnen Philosophen [wurden] streng vom Trug der Sinneswahrnehmungen und den auf bloßer Erfahrung beruhenden Meinungen der Vielen unterschieden und wesentlich höher als diese bewertet, ja, als zur Erfassung der Wahrheit und des Seins allein geeignet erachtet." (HWdP 1972: 588, vgl. auch 589-591). Die Stoiker dagegen „(...) sind der Ansicht, daß alles Denken von der sinnlichen Wahrnehmung ausgeht oder sich jedenfalls nicht ohne diese vollzieht und daß nichts auf dem Gebiete des Denkens zu finden ist, was man nicht auf Grund eines sinnlichen Eindrucks als erkannt in sich trägt." (HWdP 1972: 591). Sehr interessant scheint in dieser Hinsicht die Auffassung von Epikur zu sein, der behauptete, dass das Denken an sich kein objektives Kriterium voraussetzt, mithilfe dessen zwischen wahren und falschen Dingen unterschieden werden kann. Dagegen liegt „(...) das einzige Wahrheitskriterium (...) in

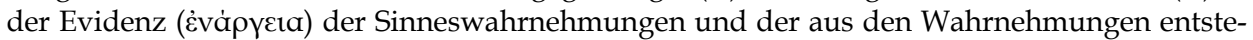

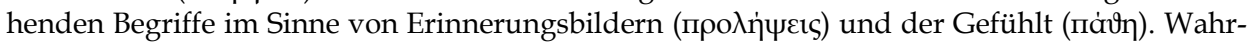
nehmungen sind immer wahr, Wahrheit und Unwahrheit liegen erst in dem von der Vernunft gefällten Urteile (...)“ (HWdP 1972: 591). 


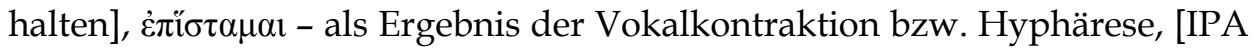
*Ep' histamai - dt. „mit etw. konfrontiert werden“, „von etw. Kenntnis nehmen“]. (vgl. Frisk 1960: 542-543). Abgesehen von der Herkunft des altgriechischen Verbs - diese lässt allerdings Schlüsse anthropologischer Art ziehen -, dessen Exegese gegenüber seines althebräischen „Äquivalents"

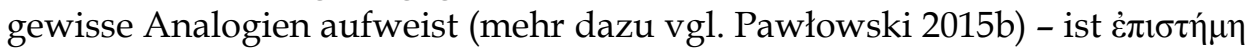
im modernen Griechischen unmittelbar auf ,Verständnis', 'Wissen' und ,Wissenschaft' $\mathrm{zu}$ beziehen.

Mit dem Designat ,(wissenschaftliches) Wissen' wird interessanterweise auch ein anderer griechischer Ausdruck, d. h. neugr. $\gamma v \tilde{\omega} \sigma ı \varsigma$ [dt. (praktische) Kenntnis] korreliert (vgl. u. a. F. Grucza 2012a). $\gamma v \tilde{\sigma} \sigma 1 \varsigma$ wurde [jedoch] im Altgriechischen als Wissen verstanden, was sich unter anderem in Genesis

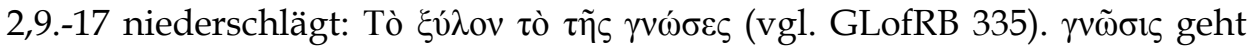
auf das altgriechische $\gamma \mathbf{v \omega ́} \sigma \kappa \omega$ [att. $\gamma 1 \gamma v \omega ́ \sigma \kappa \omega]$ zurück, was mit ,wissen', ,er-

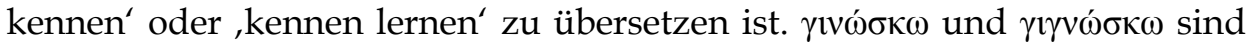

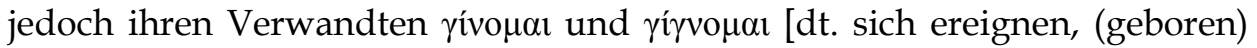
werden, entstehen, erzeugen] isomorph (vgl. Frisk 1960: 307-309) und deshalb für die hier betriebene Semantik inadäquat.

\section{DEUTUNG DES TERMINUS ,EPISTEMISCH`}

Die Diskussion über die Designate, die dem Terminus ,epistemisch' im Rahmen der Epistemologie zugeschrieben wurden oder die Polemik damit, soll hier dahingestellt bleiben. ${ }^{5}$ Im vorliegenden Beitrag geht es nicht darum, zwischen verschiedenen Arten des Wissens zu unterscheiden, d. h., zwischen dem, wie sich das Wissen als kognitive Eigenschaft manifestiert, etwa als Kenntnis, Können, Fertigkeit usw. Ich konzentriere mich dagegen auf Wissen als Erkenntnis, und zwar sowohl als Erkenntnis-Prozess wie auch als Erkenntnis-Ergebnis (vgl. S. Grucza 2012: 87-88). Diese zwei Aspekte des Forschungsgegenstandes, auf den unten detaillierter eingegangen wird, betrachte ich als den eigentlichen Schlüssel zur Aufklärung der im Rahmen der epistemologischen Semantik zu formulierenden Fragen.

Erkenntnisprozesse laufen gewöhnlich in einem ,definierten' interneren (d. h. kognitiven) Kontext ab, welchen Wissenselemente / Engramme durch und durch ausmachen. $\mathrm{Ob}$ und wenn ja, dann wie ein bestimmter internerer Kontext erkenntnisfördernd ist, bzw. sein kann, bleibt jeweils zu examinie-

5 Verwiesen sei an dieser Stelle auf einige zusammenfassende Darstellungen des Problems etwa in Handbook of Epistemology (2004) oder Powszechna encyklopedia filozofii (2002). 
ren. Stringent scheint hier wenigstens die Schlussfolgerung, dass durch den bzw. in dem interneren Kontext, und zwar mit einem "Set' von Wissenselementen, ein epistemisches Potenzial geschaffen ist, aufgrund dessen neues Wissen generiert werden kann. Mit anderen Worten, der internere Kontext, der ein logischer Teil aktuellen Wissens schlechthin ist, ermöglicht, mehr noch präsupponiert es, dass ein Erkenntnisergebnis überhaupt erlangt werden kann. Mit einem Erkenntnisergebnis, bzw. Erkenntniserfolg hat man m. E. erst dann zu tun, wenn mit einem ,Set' von bestimmten Wissenselementen, die durch epistemische Faktoren geformt sind, neues Wissen kreiert wird. Wendet man dies auf das Problem der Bedeutungskonstitution an, so ergeben sich einige grundlegende Anwendungsbereiche für die Deutung des Terminus. Mit ,epistemisch' bezeichne ich:

(1) Wissenselemente/Engramme, die als potenzielle Wissensformanten die idiolektale Ausdrucksform und die damit zu korrelierende idiolektale Bedeutung ausmachen.

(2) Wissensformanten-Typen, die sich an der Ausdrucks- und Bedeutungskonstitution beteiligen.

(3) Wissensrahmen, in dem bzw. durch den mentale Ausdrucksformen und (wirkliche) Bedeutungen ,ins Leben gerufen werden'.

(4) Als ,epistemisch' gilt außerdem die Erfahrung, dank deren, und genauer, dank der sie fundierenden sensuellen Eigenschaften, nicht nur bewusste oder unbewusste Erkenntnisakte, sondern vor allem - jedenfalls weltlich bedingte - Erkenntnisprozesse an sich überhaupt möglich sind.

Bringt man diese Anwendungsbereiche mit den gleich oben angedeuteten Annahmen in Beziehung, so ergibt sich ein Zusammenhang der Designate ,epistemisch' und ,neu'. Schlüssig werden damit die Funktionen, die den Designaten der o.g. Anwendungsbereiche zugeschrieben werden können.

(Ad. 1.) Wissenselemente/Engramme sind das kognitive Potenzial eines Individuums. Außerdem können sie je nach Erkenntnisaktivität als Wissensformanten fungieren, d. h. ihnen werden gezielte (kognitive) Funktionen in Signifikationsprozessen erteilt, sodass bereits erworbene Zeichenelemente oder völlig neue Zeichenelemente, wie die neologischen (u. z. eine idiolektale Ausdrucksform und idiolektale Bedeutung) im neuen internen Kontext oder/und angesichts des neuen externen Kontexts aktiviert bzw. generiert werden. Es sei vermerkt, dass zwischen Wissenselementen und Wissensformanten prinzipiell keine ontologische Differenz besteht. Der eigentliche Unterschied spielt sich sozusagen auf der Achse ,aktiv-passiv ${ }^{\star}$ ab. Mit den Termini ,Wissenselemente', ,Wissensformante' soll lediglich auf die in den Neurowissenschaften bereits bewiesene Tatsache hingewiesen werden, dass 
sowohl ,bloße' kognitive Akte als auch die ,außerordentlichen' Erkenntnisakte nicht nur einmalig, sondern vor allem inkomparabel sind. Anders gesagt, Wissenselemente / Engramme sind passiv, bis konkrete Neuronen feuern.

(Ad. 2.) Die Signifikation erfolgt aufgrund des bereits erworbenen oder des zu erwerbenden Potenzials. Das letztere wird durch die epistemischen Wissensformanten-Typen gewährleistet, die eine jede Ausdruck- und Bedeutungskonstitution anthropologisch, kulturell und/oder axiologisch formen. Wissensformanten-Typen ermöglichen vor allem die Herstellung neuen Wissens aber auch die kontextadäquate (intern und extern) sowie die kommunikationsadäquate Verarbeitung und Aktivierung des bereits erworbenen Wissens.

(Ad. 3.) Der (interne) Wissensrahmen setzt jede Signifikation voraus. Zeichenelemente entstehen nämlich nie in Isolation. Ihre Konstitution wird durch die Konstitution anderer Zeichenelemente bedingt. Die interne Kontextualisierung der Zeichenelemente im epistemischen (verstehensrelevanten) Wissensrahmen kann auf der Wortfeld-, Prädikations-, Satz- oder Textebene erfolgen (vgl. Busse 2007a: 6 ff., 2012, u. a. 523 f.). Der Wissensrahmen gilt als das kognitive Potenzial, dank deren Erkenntnisakte ausgeführt werden. Das will heißen, dass ein jeder Wissensrahmen potenziell erkenntnisfördernd oder, dass das Erkenntnisergebnis letztendlich auf ihn zurückzuführen ist.

(Ad. 4.) Jede Erkenntnis hat ihren kognitiven ,Hintergrund'. Mit anderen Worten, es kann keine Erkenntnis erfolgen, ohne dass eine epistemische Erfahrung zustande kommt oder/und auf eine zurückliegende epistemische Erfahrung zurückgegriffen wird. Die Sache ist nicht unkompliziert. So lassen sich zwar Erkenntnis-Akte recht unmissverständlich sowohl auf eine gegenwärtige als auch auf eine zurückliegende Erfahrung zurückführen, so ist das Erkenntnis-Ergebnis gerade in Hinblick auf die epistemischen Wissensformanten durchaus schwer nachzuvollziehen, da es an einer exakten Diagnose hinsichtlich der Proportion der sich an ihm (dem Erkenntnisergebnis) beteiligten Wissenselemente mangelt. Nichtsdestotrotz gilt die Erfahrung als epistemisch, solange sie dank der sie prägenden anthropologischen, kulturellen und/oder axiologischen Wissensformanten zu einem Erkenntnisergebnis beiträgt, welches nichts weiter ist als das neue Wissen.

\section{FRAGEN UND PROBLEME}

In diesem Lichte kommen rhetorische Fragen auf, die in Hinblick auf die oben angedeutete Insuffizienz bei der Differenzierung der Termini und De- 
signate ,epistemisch' und ,kognitiv' ertönen mögen: Sind epistemische Prozesse, epistemische Leistungen oder epistemische "Denkbewegungen“, d. h. ganz konkrete mentale Erkenntnis-Akte keine kognitiven Akte? Ist das Erkenntnis-Ergebnis kein wirkliches Ergebnis der menschlichen Kognition? Sind epistemische Wissenselemente, die in Prozessen der idiolektalen Ausdrucks- und Bedeutungskonstitution (z.B. bei der Konstitution von Fachneologismen) anthropologisch, kulturell und/oder axiologisch geformt werden, keine kognitiven? Diese Fragen sollten vor allem illustrieren, dass ,kognitiv ${ }^{\prime}$ als quasi Fachausdruck allein in der Linguistik diverse hyperonyme Funktionen aufweist, ganz zu schweigen von Designaten, die mit ,kognitiv' in Verbindung gebracht werden. Trotzdem wird der hyperonyme Gebrauch prinzipiell keine gewaltigen Probleme bereiten, solange hier über mentale Prozesse, ihre Organisation, ihre Funktionen oder Aufgaben diskutiert wird. Alles in Allem lassen sich sämtliche Denkprozesse auf die mentale Sphäre oder - wenn man so will - auf die Kognition eines Individuums zurückführen. Schließlich ,haben' die kognitiven und Erkenntnis-Akte (wohl auch auf der deskriptiven Ebene) den gleichen ontologischen Status. Problematisch wird jedoch der Unterschied zu markieren sein, mit dem ontologische von den rein funktionalen Aspekten der kognitiven Akte, und zwar der ontologische und funktionale Unterschied zwischen den Designaten ,epistemisch' und ,kognitiv', zur (Fach-)Sprache gebracht werden müssten. Bisher ist es nicht möglich, die (reinen) epistemischen Akte (Erkenntnisakte schlechthin) von den sie fundierenden kognitiven Akten (dem bis zur Zeit der Erkenntnis erworbenen und internalisierten Wissen) auf der Ebene der menschlichen Kognition empirisch auseinanderzuhalten. Möglich ist dies dagegen auf der Äußerungs- und vor allem auf der Deskriptionsebene. Gerade in der Fachsprachenforschung spielt das eine große Rolle, da jedem Terminus (jedem Fachneologismus), mit welchem ein realer und/oder konzeptueller Sachverhalt erfasst werden soll, aus Prinzip eine neue Wirklichkeit zugrunde liegt. ${ }^{6}$

\section{8. ,EPISTEMISCH`IN DER (FACHSPRACHEN-)SEMANTIK}

Auf das Problem der Erkenntnis-Funktion von Fachsprachen wiesen schon vor über einem Vierteljahrhundert im deutschsprachigen Diskurs

\footnotetext{
${ }_{6}$ Um das Problem synthetisch festzuhalten, gebrauche ich im Folgenden den Terminus ,kognitiv' in Bezug auf sämtliche Wissenselemente und -relationen, die die mentale Sphäre bis zum Zeitpunkt der Erkenntnis konstituieren. Mit dem Terminus ,epistemisch' dagegen hebe ich nеuе Erkenntnis-Prozesse, darunter nеuе Erkenntnis-Akte und neue Erkenntnis-Leistungen hervor. Kurz: kognitiv $=$ mental, hingegen epistemisch $=$ mental und vor allem erkenntnisfördernd.
} 
Dieter Möhn und Roland Pelka hin. Sie äußern sich in einer 1984 abgefassten Monografie Fachsprachen. Eine Einführung dazu folgendermaßen: „Wir verstehen unter Fachsprache heute die Variante der Gesamtsprache, die der Erkenntnis und begrifflichen Bestimmung fachspezifischer Gegenstände (...) dient (...) (zit. nach Fleischer, Helbig, Lerchner 2001: 459). Dagegen akzentuiert Franciszek Grucza im polnischen Diskurs vor allem die menschliche Erkenntnis-Fähigkeit in Hinblick auf die Konstitution und den Gebrauch von Termini. Die Termini bezeichnet er als "Mittel“, die es ermöglichen, nicht nur die Wirklichkeit bloß angemessen zu systematisieren, sondern auch all ihre feinsten Bestandteile $\mathrm{zu}$ identifizieren und $\mathrm{zu}$ differenzieren (vgl. F. Grucza 1991: 17f.). Es ist in diesem Zusammenhang sinnvoll, auf die essenzielle und durchaus unveräußerliche menschliche Eigenschaft, nämlich die epistemische Eigenschaft und Fähigkeit hinzuweisen, neue Termini/ Fachneologismen zu generieren, diese zu operationalisieren und zu evaluieren. Diese Fähigkeit resultiert aus dem Bedürfnis zu der Signifikation des neuen und der Organisation des bereits internalisierten Wissens.

Einem Terminus und insbesondere einem Fachneologismus kommen aus der Sicht der epistemologischen (Fachsprachen-)Semantik wesentliche Eigenschaften und/oder Funktionen zu.

- Mentale Äußerungsform des Terminus/Fachneologismus ist ein durchaus umfangreicher Bereich des aktuellen Wissens über die Phonemik, Graphemik, Morphemik, Grammatik, Lexik, Terminologie und Textemik der (Ziel-)Sprache (vgl. S. Grucza 2012: 135-138),

- Fachbedeutung ist ein Bereich des aktuellen Fachwissens,

- Terminus/Fachneologismus ist das Ergebnis/Fachelement der menschlichen Erkenntnis-Akte,

- Terminus/Fachneologismus ist erkenntnisfördernd - beide stimulieren die Erkenntnis, und zwar beteiligen sie sich an der Erzeugung neuen Wissens im fachsprachlichen Wissensrahmen,

- Fachneologismus ist das neue Wissen, dank dem ein Fachmann und/oder Linguist das bereits internalisierte Wissen organisiert,

- Terminus/Fachneologismus kann als oder im Text kommuniziert werden,

- die grundlegende Bedingung, Termini/Fachneologismen zu kommunizieren, ist die Fähigkeit sie zu operationalisieren, d. h. in kommunikativen / diskursiven Akten regelrecht zu gebrauchen,

- die Konstitution von Fachneologismen ist nicht arbiträr (vgl. auch Pawłowski 2015b). 


\section{FAZIT}

Diese vorläufigen Thesen mögen illustrieren, dass die Unterscheidung zwischen ,kognitiv' und ,epistemisch' auf der konzeptuellen, terminologischen und deskriptiven Ebene durchaus relevant ist. Um das Problem auf den Punkt zu bringen, kann folgendes gesagt werden: Kognitive Prozesse sind keine epistemischen, solange sie nicht einen deutlichen erkenntnismäßigen, -fördernden Status haben. Epistemische Akte sind relativ (!) Ihr Status hängt davon ab, ob bzw. in welchem Maße den kognitiven Akten ErkenntnisFunktion(en) ,beigemessen' werden. Das heißt, nicht alle kognitiven Akte sind gleich erkenntnisfördernd. So ist zum Beispiel das Sehen, Hören, Tasten, Riechen, etc., sogar Reflektieren, zwar kognitiv bedingt, und zwar wir sehen, hören, tasten, riechen und reflektieren mit und in dem Gehirn, so ist der Erkenntnis-Status der Wissenselemente, die sich im Gefolge der kognitiven Prozesse des Sehens, Hörens, Tastens, Riechens, Reflektierens usw. herausbilden, noch lange nicht gewährleistet. Ob Wissens-Elemente als epistemische Wissens-Formanten in die Erkenntnisakte, darunter in die Akte der Ausdrucks- und Bedeutungskonstitution von (Fach-)Neologismen aufgenommen werden, hängt jeweils vom Erkenntnis-Potenzial eines konkreten Fachmanns/Linguisten ab, der sich zum Ziel setzt, einen neuen Fachbereich zu identifizieren, zu signifizieren bzw. zu organisieren. Worauf er sich dabei bezieht, d. h., was seinen Erkenntnis-Akten zugrunde liegt, gehört meines Erachtens zu den grundlegendsten Problemen und Aufgaben der epistemologischen Semantik.

\section{LITERATURVERZEICHNIS}

Bock von Wülfingen, B., 2010. Epistemologie und Differenz. Zur Reproduktion des Wissens in den Wissenschaften. Bielefeld: Transcript.

Busse, D., 1992. Textinterpretation: Sprachtheoretische Grundlagen einer explikativen Semantik. Opladen: Westdeutscher Verlag.

Busse, D., 2005. Architekturen des Wissens - Zum Verhältnis von Semantik und Epistemologie. In: Müller E. (Hrsg.). Begriffsgeschichte im Umbruch. (= Archiv für Begriffsgeschichte, Sonderheft 2004) Berlin: Felix Meiner, 43-57, <http://www.phil-fak.uniduesseldorf.de/fileadmin/Redaktion/Institute/Germanistik/AbteilungI/Busse/ Texte/Busse-2005-03.pdf>, [letzte Einsicht am 15.05.2015].

Busse, D., 2007a. Diskurslinguistik als Kontextualisierung - Sprachwissenschaftliche Überlegungen zur Analyse gesellschaftlichen Wissens. In: Warnke I. (Hrsg.). Diskurslinguistik nach Foucault. Berlin: De Gruyter, 81-105, <http://www.phil-fak.uniduesseldorf.de/fileadmin/Redaktion/Institute/Germanistik/AbteilungI/Busse/ Texte/Busse-2007-01-ur.pdf>, [letzte Einsicht am 15.05.2015]. 
Busse, D. 2008a. Diskurslinguistik als Epistemologie. Das verstehensrelevante Wissen als Gegenstand linguistischer Forschung. In: Warnke I., Spitzmüller J. (Hrsg.). Methoden der Diskurslinguistik. Sprachwissenschaftliche Zugänge zur transtextuellen Ebene. Berlin, 57-88, <http://www.phil-fak.uni-duesseldorf.de/fileadmin/Redaktion/Institute/Ger manistik/AbteilungI/Busse/Texte/Busse-2008-03-ur.pdf>, [letzte Einsicht am 15.05.2015].

Busse, D., 2008b. Begriffsgeschichte - Diskursgeschichte - Linguistische Epistemologie. Bemerkungen $\mathrm{zu}$ den theoretischen und methodischen Grundlagen einer Historischen Semantik in philosophischem Interesse anlässlich einer Philosophie der Person. In: Haardt A., Plotnikov N. (Hrsg.). Diskurse der Personalität: Die Begriffsgeschichte der ,Person' aus deutscher und russischer Perspektive. München: Fink, 115-142, <http://www.phil-fak.uni-duesseldorf.de/fileadmin/Redaktion/Institute/Germa nistik/AbteilungI/Busse/Texte/Busse-2008-04-ur.pdf $>$, [letzte Einsicht am 15.05.2015].

Busse, D., 2008c. Linguistische Epistemologie. Zur Konvergenz von kognitiver und kulturwissenschaftlicher Semantik am Beispiel von Begriffsgeschichte, Diskursanalyse und Frame-Semantik. In: Kämper H., Eichinger L. (Hrsg.). Sprache - Kognition - Kultur. Sprache zwischen mentaler Struktur und kultureller Prägung. (= Jahrbuch 2007 des Instituts für deutsche Sprache) Berlin/New York: De Gruyter, 73-114, <http:// www.phil-fak.uni-duesseldorf.de/fileadmin/Redaktion/Institute/Germanistik/ AbteilungI/Busse/Texte/Busse-2008-02-ur.pdf $>$, [letzte Einsicht am 15.05.2015].

Busse, D., 2008d. Semantische Rahmenanalyse als Methode der Juristischen Semantik. Das verstehensrelevante Wissen als Gegenstand semantischer Analyse. In: Christensen R., Pieroth B. (Hrsg.). Rechtstheorie in rechtspraktischer Absicht. Freundesgabe zum 70. Geburtstag von Friedrich Müller. (= Chriften zur Rechtssprache 235) Berlin: Dunckler \& Humbolt 35-55, <http://www.phil-fak.uni-duesseldorf.de/fileadmin/ Redaktion/Institute/Germanistik/AbteilungI/Busse/Texte/Busse-2008-01-ur.pdf>, [letzte Einsicht am 15.05.2015].

Busse, D., 2012. Frame-Semantik. Ein Kompendium. Berlin/Boston: De Gruyter.

Devitt, M., 2012a. Semantic Epistemology: Response to Machery. Theoria, 74, 229-233.

Devitt, M., 2012b. Whither Experimental Semantics. Theoria, 73, 5-36.

Fleischer, W., Helbig, G., Lerchner, G. (Hrsg.), 2001. Kleiner Enzyklopädie Deutsche Sprache. Frankfunt/M: Peter Lang.

Frisk, H., 1954-1972. Griechisches Etymologisches Wörterbuch. Heidelberg, Bd. 1., 1-XXX, 1938 [а-ко] 1960; Bd. 2., 1-1154 [к $\rho-\omega]$ 1970; Bd. 3., 1-312 [Nachträge, Wortregister, Corrigenda] (1972), <http://ieed.ullet.net/friskL.html>, [letzte Einsicht am 15.05.2015].

Fumerton, R., 1994. Skepticism and Naturalistic Epistemology. In: French P., Uehling Th., Wettstein H. (Hrsg.). Midwest Studies in Philosophy XIX. Notre Dame: University of Notre Dame Press, 321-340.

Gabriel, M., 2008. An den Grenzen der Erkenntnistheorie. Die notwendige Endlichkeit des objektiven Wissens als Lektion des Skeptizismus. München: Karl Alber.

(GLofRB) Greek Lexicon of the Roman and Byzantine Period from B.C. 146 to A.D. 1100, 1900. Sophocles E.A. (Hrsg.), New York: Charles Scribner's Sons.

Grucza, F., 1991. Terminologia. Jej przedmiot, status i znaczenie. In: Grucza F. (Hrsg.). Teoretyczne podstawy terminologii. Seria Komitetu Terminologii. Problemy Terminologii 1. Wrocław, Warszawa, Kraków: Zakład Narodowy im. Ossolińskich, 11-44. 
Grucza, F., 1994. Języki specjalisyczne - ich rozumienie i znaczenie. In: ders., Kozłowska Z. (Hrsg.). Jezzyki specjalistyczne. Materiaty XVIII Sympozium ILS UW (Warszawa, 9-11 stycznia 1992), Warszawa: Wydawnictwo Akapit, 7-27.

Grucza, F., 2012a. Kulturologia antropocentryczna a kulturoznawstwo. In: Grzywka K., Filipowicz M., Godlewicz-Adamiec J., Jagłowska A., Kociumbas P., Małecki R., Michta E., Wyrzykiewicz D. (Hrsg.). Kultura - Literatura - Jezyk/Kultur - Literatur Sprache, Bd. 1., Warszawa: IG UW, 79-102.

Grucza, F., 2012b. Zum Gegenstand und zu den Aufgaben der anthropozentrischen Linguistik, Kultlturologie und Kommunikologie sowie zur gegenseitigen Vernetzung dieser Erkenntnisbereiche. Kwartalnik Neofilologiczny, LIX, 3, 287-344.

Grucza, S., 2012. Fachspachenlinguistik. Warschauer Studien zur Germanistik und Angewandter Linguistik 1. Frankfurt/M.: Peter Lang.

Grucza, S., 2013. Jezyk a poznanie - kilka uwag na temat rozważania ich wzajemnych relacji. In: ders., Marchwiński A., Płużyczka M. (Hrsg.). Translatoryka. Koncepcje - Modele - Analizy. Księga jubileuszowa ofiarowana Profesor Barbarze Z. Kielar z okazji 80. rocznicy urodzin. Warszawa: Wydawnictwo Naukowe IKLA WU, 54-67.

Handbook of Epistemology (HE), 2004, herausgegeben von Ilkka Niiniluoto, Matti Sintonen und Jan Woleński, Dordrecht, Boston, London: Kluwer Academic Publishers.

Historisches Wörterbuch der Philosophie (HWdP), 1972, herausgegeben von Joachim Ritter, völlig neu. bearbeit. Ausg. von Rudolf Eisler, Bd. 2., Basel, Stuttgart.

Künne, W., 2007. Abstrakte Gegenstände. Semantik und Ontologie. Frankfurt/M.: Klostermann.

Machery, E., 2012. Semantic Epistemology: A Brief Response to Devitt. Theoria, 74, 223227.

Möhn, D., Pelka, R., 1984. Fachsprachen. Eine Einführung. Tübingen: Francke.

Moore, R., 1983. Semantical considerations on nonmonotonic logic. In: Bundy A. (Hrsg.). Proceedings of the $8^{\text {th }}$ International Joint Conference on Artifical Intelligence. Karlsruhe: William Kaufmann, 272-279.

Moser, F., 2012. Subjekt - Objekt - Intersubjektivität. Eine Untersuchung zur erkenntnistheoretischen Subjekt - Objekt - Dialektik Hegels und Adornos mit einem Ausblick auf das Intersubjektivitätsparadigma Habermas'. Bern/Berlin/Bruxelles/Frankfurt/M./New York/ Oxford/Wien: Peter Lang.

Quine, W. v. O., 1969. Epistemology Naturalized. In: Quine W. v. (Hrsg.). Ontological Relativity and other Essays. New York: Columbia University Press, 69-90.

Quine, W. v. O., 1981. The Nature of Natural Knowledge. In: French P. (Hrsg.). Foundations in Analytic Philosophy. Minneapolis, 67-81.

Quine, W. v. O., 1995. Naturalism; Or, Living Within One's Means. Dialectica, 49, 251-261.

Pawłowski, G., 2015a. Ein Plädoyer für die epistemologische Semantik. Einige Überlegungen zur Konzeption, zu den Aufgaben und zur Analyse der FachtextÄußerungen aus dem Bereich , altgriechische Musik'. Glottodidactica, 2/2015, i.D.

Pawłowski, G., 2015b. ,auleta' und es bleibt alles beim Neuen!? Zur Konstitution der polnischen Fachneologismen im Bereich der altgriechischen Musik. Zeitschrift des Verbandes Polnischer Germanisten, 1/2015, 45-63.

Pawłowski, G., 2015c. Translatorische Aspekte bei der Konstitution von polnischen Fachneologismen im Bereich, altgriechische und byzantinische Musik'. In: Weigt Z. (Hrsg.), Felder der Sprache - Felder der Forschung. Lodzer Germanistikbeiträge III, Bd. 2., Łódź: Wydawnictwo Primum Verbum, i.D. 
Plümacher, M., 2004. Wahrnehmung, Repräsentation und Wissen. Edmund Husserls und Ernst Cassirers Analysen zur Struktur des Bewusstseins. Berlin: Parerga-Verlag.

Powszechna encyklopedia filozofii (PEF), 2002. Krąpiec M., Lobato A., Maryniarczyk A., Jaroszyński P., Kiereś H., Zdybicka Usjk, Z.J. (Hrsg.), Lublin: Polskie Towarzystwo Tomasza z Akwinu.

Stownik grecko-polski (SłGP), 1960. Abramowiczówna Z. (Hrsg.), Warszawa: PWN.

Shoham, Y., 1987. Nonmonotonic logics: meaning and utility. In: Kaufmann M. (Hrsg.). Proceedings of the 10 International Joint Conference on Artifical Intelligence. Milan: Morgan Kaufman, 388-393.

Skowronek, B., 2009. Epistemologische Begründung für den gesellschaftlichen Status des Fremdsprachenunterrichts. Scripta Neophilologica Posnaniensia, 10, 127-137. 
